\title{
Luminosity, selfgravitation and nonuniqueness of stationary accretion
}

\author{
J. Karkowski ${ }^{1}$, E. Malec ${ }^{1,2}$, and K. Roszkowski ${ }^{1}$ \\ 1 M. Smoluchowski Institute of Physics, Jagiellonian University, Reymonta 4, 30-059 Kraków, Poland \\ e-mail: malec@th.if.uj.edu.pl \\ 2 Max Planck Institute for Gravitational Physics (AEI), Am Mühlenberg 1, 14-476 Potsdam, Germany
}

Received 3 July 2007 / Accepted 24 October 2007

\section{ABSTRACT}

\begin{abstract}
Aims. We show the existence of two branches of solutions bifurcating from a point with maximal luminosity. Methods. We investigate a Newtonian description of accreting compact bodies with hard surfaces, including luminosity and selfgravitation of polytropic perfect fluids. This nonlinear integro-differential problem is studied numerically. Its reduced version simplifies (under appropriate boundary conditions) to an algebraic relation between luminosity and the gas abundance in stationary, spherically symmetric flows and it can be dealt with analytically.

Results. There exist - for a given luminosity, asymptotic mass and asymptotic temperature - two sub-critical solutions that bifurcate from an extremal point. They differ by the fluid content and the mass of the compact centre. Their relevance to Thorne-Żytkow stars is discussed.
\end{abstract}

Key words. accretion, accretion disks - hydrodynamics - gravitation - instabilities

\section{Introduction}

In this paper we study a Newtonian accreting systems consisting of a compact body enclosed in a ball of selfgravitating gas. Our aim is to investigate the following inverse problem: assume that one knows the luminosity, total mass, asymptotic temperature (or, equivalently, the asymptotic speed of sound) and the equation of state of the gas. Assume that the gravitational potential has a fixed value at the boundary of a compact body which can be interpreted as fixing gravitational redshift of radiation emitted from the surface. The question arises: can one specify the mass of the compact body? And if one can, is the specification unique?

There are two contexts in which that issue arises. Firstly, it relates to the question of identification of Thorne-Żytkow stars (TŻS hereafter) (Thorne \& Żytkow 1977), that could be formed by the merger of a main sequence star with a neutron star or a black hole. They can be powered mainly by sub-Eddington gas accretion, if their gas envelope is sufficiently large, and emit electromagnetic radiation. The other hypothetical TŻS, with dense atmospheres, super-Eddington accretion (Chevalier 1993) and radiating mainly in neutrinos, are not discussed in this paper.

Secondly, there is the question of whether observations can distinguish compact bodies with a hard surface (neutron stars or recently proposed gravastars, Mazur \& Mottola 2004) from black holes. Broderick and Narayan (Broderick \& Narayan 2006) presented observational arguments in favour of the existence of black holes. Abramowicz et al. (2002) raised several objections, expressing their view that present accretion disk models are not precise enough. It is known that some gravitational wave signals (quasinormal modes) are distinctly different for the two classes of objects and that issue can hopefully be solved when the gravitational wave detectors begin to collect astronomical data.
We choose below the simplest self-contained model that can be interpreted as a radiating system. Our results apply primarily to Thorne-Żytkow stars. We show that there is an ambiguity even in the Newtonian level. There can exist at least two solutions with a hard surface - two compact stars (TŻSs, for instance) - to the inverse problem described above. This is not unexpected. In a recent general-relativistic analysis of selfgravitating perfect fluids (valid also in the Newtonian limit) the mass accretion rate $\dot{M}$ behaves like $y^{2}(1-y)$, where $y=M_{*} / M$ is the ratio of (roughly) the mass of the compact core to the total mass (Karkowski et al. 2006). Thus there exists a maximum of $\dot{M}$ when $y=2 / 3$. The luminosity equals mass accretion rate $\dot{M}$ multiplied by the available energy per unit mass the potential $\phi\left(R_{0}\right)$ at the hard surface of a compact star. Hence this simple analysis of accretion would suggest the existence of two weakly luminous regimes: one rich in fluid with $m_{\mathrm{f}} \approx M$ and the other with a small amount of fluid (test fluid approximation), $m_{\mathrm{f}} / M \ll 1$. To a given luminosity $x$ might have corresponded two systems with different gas abundances $1-y$. The situation considered below, with the luminosity taken into account, is less clear. It is shown below that the radiation also impacts the accretion rate $\dot{M}$ and there emerges a complicated algebraic relation $y=y(x)$. Nevertheless, the nonuniqueness of solutions still appears.

\section{The Shakura model}

Stationary accretion of spherically symmetric fluids with luminosities close to the Eddington limit has been investigated since the pioneering work of Shakura (1974). There, the gas pressure and its selfgravity have been ignored and the analysis is purely Newtonian. The work of Buff \& McCray (1974) is dedicated to a similar problem. Later researchers included both gas pressure and selfgravity (Okuda \& Sakashita 1977; Maraschi et al. 1978; Chia 1978, 1979; Grindlay 1978). In Chia (1978) - in 
which radiation is absent - it was shown that selfgravitation affects some parameters (radius and mass) of the sonic point; that agrees with Karkowski et al. (2006) (but there the analysis was general-relativistic, in the contrast to Chia 1978). Another conclusion of Chia (1978), that selfgravity increases the mass accretion rate, is true only if the gas contributes less than $1 / 3$ of the total mass of the system; for greater abundances the mass accretion rate decreases (Karkowski et al. 2006). Some quantitative results of Chia (1978) and Chia (1979) can be misleading since there is a mistake in the energy conservation law (see formula (2.3) in Chia 1978). The analysis was extended later to general-relativistic systems (Thorne et al. 1981; Park \& Miller 1991; Rezzolla \& Miller 1994).

We will consider a spherically symmetric compact ball of a fluid falling onto a non-rotating compact body. The radial velocity $U$ of a comoving particle labelled by coordinates $(r, t)$ is given by $U(r, t)=\partial_{t} R$, where $t$ is a comoving (Lagrangian) time. $p$ denotes pressure, $L(R)$ and $L_{\mathrm{E}}$ are the local and critical (Eddington) luminosities, respectively. The quasilocal mass $m(R)$ is defined by $\partial_{R} m(R)=4 \pi R^{2} \varrho$. The mass accretion rate is

$\dot{M}=-4 \pi R^{2} \varrho U$.

$\varrho$ is the baryonic mass density. The equation of state is $p=K \varrho^{\Gamma}$ with $\Gamma$ being a constant, $(1<\Gamma \leq 5 / 3)$. The total mass $m\left(R_{\infty}\right)$ will be denoted by $M$. We assume a steady collapse of the fluid, which means that all its characteristics are constant at a fixed areal radius $R$ : $\left.\partial_{t} X\right|_{R=\text { const. }}=\left(\partial_{t}-\left(\partial_{t} R\right) \partial_{R}\right) X=0$, where $X=\varrho, U, a^{2} . a=\sqrt{\partial_{\varrho} p}$ is the speed of sound. Strictly, a stationary accretion must lead to the increase of the central mass. This in turn means that the notion of the "steady accretion" is approximate - it demands that the mass accretion rate is small and the observation time short in comparison to the characteristic timescale $\tau=M / \dot{M}$, so that the quasilocal mass $m(R)$ does not change significantly. Furthermore, we assume that the radius $R_{\infty}$ of the ball of fluid and other boundary data are such that

$U_{\infty}^{2} \ll \frac{G m\left(R_{\infty}\right)}{R_{\infty}} \ll a_{\infty}^{2}$.

The first inequality can be interpreted as demanding that external layers of gas are so gently injected into the system from outside that they freely self-gravitate with a very small initial velocity. The second inequality relates the asymptotic values of the escape velocity and the speed of sound. The steadily accreting gas is described by a system of integro-(ordinary)-differential equations. They consist of the (Euler) momentum conservation equation

$U \partial_{R} U=-\frac{G M(R)}{R^{2}}-\frac{1}{\varrho} \partial_{R} p+\alpha \frac{L(R)}{R^{2}}$,

the mass conservation

$\partial_{R} \dot{M}=0$,

and the energy conservation

$L_{0}-L(R)=\dot{M}\left(\frac{a_{\infty}^{2}}{\Gamma-1}-\frac{a^{2}}{\Gamma-1}-\frac{U^{2}}{2}-\phi(R)\right)$.

The constant $\alpha$ is defined by $\alpha=\sigma /\left(4 \pi m_{\mathrm{p}} c\right)$ ( $\sigma$ is the Thomson cross section for scattering of radiation by free electrons) and $L_{0}$ is the total luminosity. $\phi(R)$ is the Newtonian gravitational potential,

$\phi(R)=-G \frac{M(R)}{R}-4 \pi G \int_{R}^{R_{\infty}} r \varrho(r) \mathrm{d} r$
$M(R) \equiv M-4 \pi \int_{R}^{R_{\infty}} r^{2} \varrho(r) \mathrm{d} r$ is the mass contained within the sphere $R$.

Strictly, in Eq. (5) should appear $a_{\infty}^{2} /(\Gamma-1)+U_{\infty}^{2} / 2-G M / R_{\infty}$ but this is well approximated by $a_{\infty}^{2} /(\Gamma-1)$, due to the boundary conditions (2).

We define the Eddington luminosity as that at the outermost layer of the accreting gas, that is as $L_{\mathrm{E}}=G M / \alpha$, while the total luminosity $L_{0}$ is equal to the product of the mass accretion rate and the total available energy per unit mass $\phi_{0} \equiv\left|\phi\left(R_{0}\right)\right|$, where $R_{0}$ is an areal radius of the boundary of the compact body, $L_{0}=\dot{M} \phi_{0}$ (Shakura 1974). This means that the total gravitational binding energy is converted into outgoing radiation. Therefore it is reasonable to neglect the outflow of gas particles that are reflected from the surface and to restrict our attention to the accretion branch which - similarly to the Bondi model (Bondi 1952 ) - is one of the four emerging mathematical possibilities.

The quantity $\phi_{0}$ is fixed and $R_{0}$ in the constructed configurations is by definition the area radius at which the absolute value of the surface potential equals $\phi_{0}$. Differentiating Eq. (5) with respect to $R$, using the equality $\partial_{R} a^{2} /(\Gamma-1)=a^{2} \partial_{R} \ln \varrho=\partial_{R} p / \varrho$ and combining the obtained equation with Eq. (3) yields the differential equation $\partial_{R} \ln L=\alpha \dot{M} / R^{2}$. Its solution can be written in terms of the Eddington and total luminosities as follows

$L=L_{0} \exp \left(\frac{-L_{0} \tilde{R}_{0}}{L_{\mathrm{E}} R}\right)$

it has the same form as in the case of test fluids (Shakura 1974). Here $\tilde{R}_{0} \equiv G M /\left|\phi\left(R_{0}\right)\right|$ is modified size measure of the compact body. In the case of test fluids $\tilde{R}_{0}=R_{0}$.

\section{The mass accretion rate}

In what follows it is assumed that there exists a sonic point, but we believe that the same (nonuniqeness) result would have been obtained for subsonic solutions. The sonic horizon (sonic point) is a location where $|U|=a$. In the following we will denote by the asterisk all values referring to the sonic points, e.g., $a_{*}$, $U_{*}$, etc. Differentiation with respect the areal radius $R$ will be denoted as prime '. One finds from the mass conservation $U^{\prime}=$ $-U\left(\left(\varrho^{\prime} / \varrho\right)+2 / R\right)$. Inserting that into the Euler Eq. (3), one finds

$\frac{\varrho^{\prime}}{\varrho}\left(a^{2}-U^{2}\right)=\frac{2}{R}\left(U^{2}-\frac{G M(R)}{2 R}+\frac{L(R) \alpha}{2 R}\right)$,

thus at the sonic point the three characteristics, $a_{*}, U_{*}$, and $M_{*} / R_{*}$ are related,

$a_{*}^{2}=U_{*}^{2}=\frac{G M_{*}}{2 R_{*}}\left(1-\frac{L_{*} \alpha}{G M_{*}}\right)=\frac{G M_{*}}{2 R_{*}}\left(1-\frac{L_{*} M}{L_{\mathrm{E}} M_{*}}\right)$.

It is clear from Eq. (9) that in order to have a critical flow one should have

$\frac{L_{*} M}{L_{\mathrm{E}} M_{*}}<1$.

It is useful to express the velocity $U$ and the mass density $\varrho$ in the following way

$U=U_{*} \frac{R_{*}^{2}}{R^{2}}\left(\frac{a_{*}^{2}}{a^{2}}\right)^{1 /(\Gamma-1)}$,

where $U_{*}$ is the negative square root, and

$\varrho=\varrho_{\infty}\left(\frac{a}{a_{\infty}}\right)^{2 /(\Gamma-1)}$, 
where the constant $\varrho_{\infty}$ is equal to the mass density of collapsing fluid at the boundary $R_{\infty}$. There exist four stationary branches crossing at the sonic point, one of which is the accretion flow.

Define

$x \equiv \frac{L_{0}}{L_{\mathrm{E}}}, \quad y \equiv \frac{M_{*}}{M}, \quad \gamma \equiv \frac{\tilde{R}_{0}}{R_{*}}$,

$\Delta_{*} \equiv L_{0}-L_{*}-2 \dot{M} a_{*}^{2} \frac{L_{*}}{y L_{\mathrm{E}}-L_{*}}$,

$\Psi_{*} \equiv-\phi_{*}-\frac{G M_{*}}{R_{*}}$.

In new variables the necessary condition (10) for the existence of a sonic point reads $x \exp (-x \gamma)<y$. Straightforward algebra leads to the following equation

$a_{\infty}^{2}-\frac{5-3 \Gamma}{2} a_{*}^{2}=(\Gamma-1)\left(\frac{\Delta_{*}}{\dot{M}}-\Psi_{*}\right)$.

We shall assume that $\gamma<1$ and $x \gamma \ll 1$. From Eq. (9)

$U_{*}^{2}=\frac{G M_{*}}{2 R_{*}}\left(1-\frac{x}{y} \exp (-x \gamma)\right)$.

Then $\Delta_{*}=0$ up to the first order while $\Psi_{*}=4 G \pi \int_{R_{*}}^{R_{\infty}} r \varrho \mathrm{d} r$. One can show, using arguments similar to those applied later, that $\left|\Psi_{*}\right| \ll a_{*}^{2}$. Therefore Eq. (14) simplifies to

$\frac{a_{*}^{2}}{a_{\infty}^{2}}=\frac{2}{5-3 \Gamma}$

that is the same result as in the Bondi model (Bondi 1952). The above derivation requires that $\Gamma$ is somewhat smaller than $5 / 3$.

The rate of the mass accretion $\dot{M}$ given by Eq. (1) can be expressed in terms of the characteristics of the sonic point. One obtains, using Eqs. (9) and (1), that

$\dot{M}=G^{2} \pi M^{2} \frac{\varrho_{\infty}}{a_{\infty}^{3}}(y-x \exp (-x \gamma))^{2}\left(\frac{a_{*}^{2}}{a_{\infty}^{2}}\right)^{(5-3 \Gamma) /(2(\Gamma-1))}$.

Now, one can can show in a way similar to that applied in the case of relativistic accretion (Karkowski et al. 2006) that under the previously assumed conditions and $R_{*} \gg \tilde{R}_{0}$ one has $\varrho_{\infty}=$ $\chi_{\infty}\left(M-M_{*}\right)=M \chi_{\infty}(1-y)$ for $\Gamma \in(1,5 / 3-\delta)$ with some small $\delta$.

More specifically, one needs to show that the energy conservation Eq. (5) yields

$\frac{a^{2}(R)}{a_{\infty}^{2}}<1-(\Gamma-1) \frac{\phi(R)}{a_{\infty}^{2}} ;$

bounding $|\phi(R)|$ from above by $G M / R$ one obtains

$\frac{a^{2}(R)}{a_{\infty}^{2}}<1+(\Gamma-1) \frac{G M}{R a_{\infty}^{2}}$.

Therefore Eq. (12) implies $\varrho \leq \varrho_{\infty}\left(1+(G M) /\left(R a_{\infty}^{2}\right)\right)^{1 /(\Gamma-1)}$. The conclusion follows now from using this bound in $M-M_{*}=$ $\int_{V} \varrho \mathrm{d} V$ and appealing to the asymptotic conditions laid down below. The proportionality constant $\chi_{\infty}$ is roughly the inverse of the volume of the gas outside of the sonic sphere. Since the total luminosity $L_{0}=\phi\left(R_{0}\right) \dot{M}$ and $a_{*}^{2} / a_{\infty}^{2}$ is given by Eq. (16), one arrives at

$$
\begin{aligned}
L_{0}= & \phi_{0} G^{2} \pi \frac{\chi_{\infty} M^{3}}{a_{\infty}^{3}}(1-y)(y-x \exp (-x \gamma))^{2} \\
& \times\left(\frac{2}{5-3 \Gamma}\right)^{(5-3 \Gamma) /(2(\Gamma-1))} .
\end{aligned}
$$

The above construction works if a solution satisfies (2); the integro-differential problem reduces to an algebraic equation. Numerical examples of Sect. 5 suggest that the set of solutions that satisfy these boundary conditions is not empty. We believe that the nonuniqueness of solutions discovered by us will manifest in a much larger set of steadily accreting systems which do not necessarily satisfy (2) and that cannot be reduced to an algebraic problem; that it can be generic. The restriction to systems obeying conditions (2) was made only in order to simplify the analysis.

\section{The analysis of the luminosity equation}

It is convenient to cast the formula (20) in terms of the relative luminosity, $x=L_{0} / L_{\mathrm{E}}$,

$x=\beta(1-y)(y-x \exp (-x \gamma))^{2}$,

where $\beta=\chi_{\infty} \phi_{0} \alpha G \pi\left(M^{2} / a_{\infty}^{3}\right)(2 /(5-3 \Gamma))^{(5-3 \Gamma) /(2(\Gamma-1))}$.

We shall analyze solutions of this cubic (with respect to the variable $y$ ) equation. One can show that there exist at least two solutions $y(x, \beta)$ for any parameter $\beta, 0 \leq \gamma<1$ and the relative luminosity $x$ is smaller than a certain extremal value $a$. We add the adjective "extremal" to all characteristics of this extremal point $(a, y(a))$.

Theorem Define $F(x, y) \equiv x-\beta(1-y)(y-x \exp (-x \gamma))^{2}$. Then

i) there exists an extremal point $x=a, y=b$ such that $F(a, b)=$ 0 and $\left.\partial_{y} F(x, y)\right|_{a, b}=0$. Here $a$ and $b$ satisfy inequalities $0<$ $a<b<1$ and $b=[2+a \exp (-a \gamma)] / 3, a=4 \beta(1-b)^{3}$;

ii) for any $0<x<a$ there exist two solutions $y(x)_{-}^{+}$bifurcating from $(a, b)$. They are locally approximated by formulae

$$
y_{-}^{+}=b \pm \frac{\sqrt{(a-x)(b+a \exp (-a \gamma)(1-2 a \gamma))}}{\sqrt{\beta(b-a \exp (-a \gamma))(1-a \exp (-a \gamma))}} .
$$

iii) the relative luminosity $x$ is extremized at the point $(a, b)$.

The proof is moved to the Appendix.

Figure 1 shows two solutions bifurcating from an extremal point in the case of $\gamma=0$ and $\beta=50$. The coordinates $a, b$ of the extremal point increase with the increase of the parameter $\beta$. Indeed, from Eq. (A.2) in the Appendix A follows $\mathrm{d} a / \mathrm{d} b=\exp (-a \gamma)(1-a \gamma)$; this is greater than zero, since $a \gamma<1$. Differentiation of the first equation in (A.2) with respect to $\beta$ yields $\mathrm{d} b / \mathrm{d} \beta=4(1-b)^{3} /\left(\mathrm{d} a / \mathrm{d} b+12 \beta(1-b)^{2}\right)>0$; we exploited here the fact that $\mathrm{d} a / \mathrm{d} b>0$. Since along the extremal curve $\mathrm{d} a / \mathrm{d} \beta=(\mathrm{d} a / \mathrm{d} b)(\mathrm{d} b / \mathrm{d} \beta)$, we obtain also $\mathrm{d} a / \mathrm{d} \beta>0$. In the particular case of $\gamma=0$ one can explicitly find $b$ by solving Eq. (A.3) (see Appendix A),

$b=1-\frac{\left(\beta^{2}+\beta^{3 / 2} \sqrt{1+\beta}\right)^{2 / 3}-\beta}{2 \beta\left(\beta^{2}+\beta^{3 / 2} \sqrt{1+\beta}\right)^{1 / 3}}$.

Equation (A.2) then gives $a(\beta)$ and one can check explicitly that both $a$ and $b$ monotonically increase with the increase of $\beta$.

An interesting fact is that at extremal points the parameter $b$ is not smaller than $2 / 3$. This lower bound is saturated at small $\beta$, i.e., when the relative luminosity $a$ is small (notice that $a<\beta$ ), which corresponds to the maximal rate of the mass accretion in irradiating systems (Karkowski et al. 2006). Since the fluid abundance is equal to $1-b$, we can conclude that extremal configurations have less fluid than $1 / 3$ of the total mass, and the upper 
Bifurcation point $=0.570,0.8258616873$

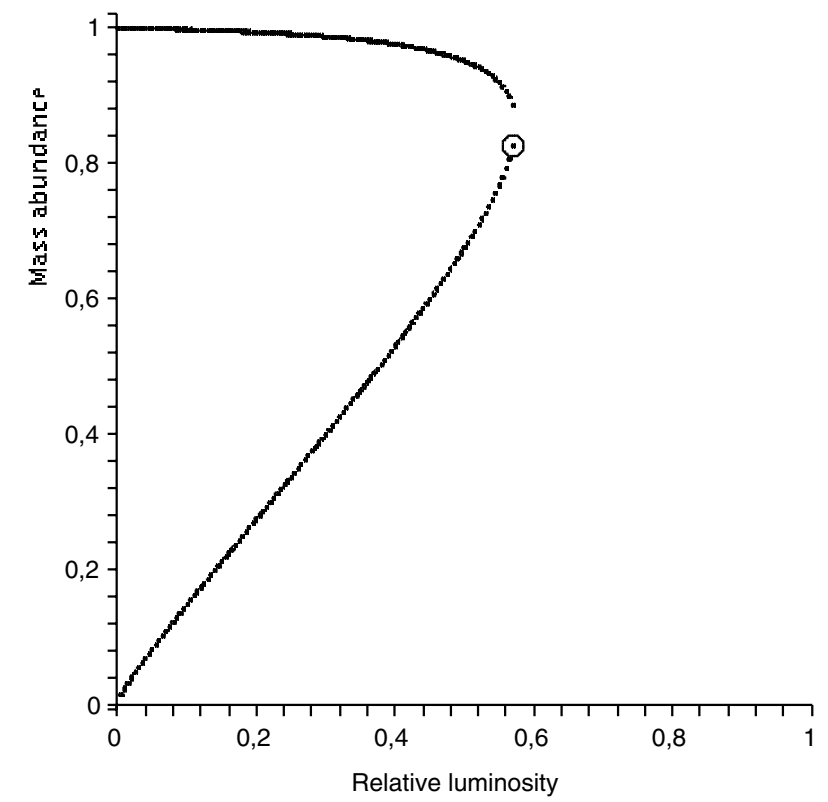

Fig. 1. The relative mass parameter $y$ (the abundance of the gas is $1-$ $y$ ) is on the ordinate while the relative luminosity $x$ is shown on the abscissa. The circle encloses the bifurcation point $(a, b)$.

bound $1 / 3$ is saturated at the limit of vanishing radiation. The maximal mass accretion rate does not occur exactly at $y=2 / 3$, as in systems with no radiation (Karkowski et al. 2006), but at a somewhat larger value.

Under a simplifying assumption that $\gamma \ll 1$ one can neglect the exponential term in $x \exp (-x \gamma)$. The analysis of Eqs. (A.2) and (23) allows us to conclude that $a$ can be as close as one wishes to 1 for large $\beta$. Putting that in physical terms, the luminosity $L_{0}$ can be close to the Eddington luminosity $L_{\mathrm{E}}$ if the parametr $\beta$ is large enough, or more precisely, if the product $\left(M / M_{0}\right)^{2} /\left(a_{\infty} / a_{0 \infty}\right)^{3}$ (where $M_{0}$ and $a_{0 \infty}$ are some reference quantities) is large enough. The two bifurcation solutions have a luminosity $x<a$ and they are characterized by two different numbers $y_{1}, y_{2}$; the latter can be markedly different (say $y_{1}>10 y_{2}$ ) only for $x \ll a$. This is suggested by point ii) of the Theorem which shows the branching of the two solutions from an extremal point, but numerical calculations confirm that more precisely. A practical conclusion is as follows: if a given system radiates with a luminosity close to its extremal luminosity (and, in particular, to the Eddington limit), then the cores corresponding to the bifurcating solutions have similar masses. The necessary and sufficient conditions for having two cores with significantly differing masses, $M_{1} \ll M_{2}$, is that the luminosity is much smaller than $L_{\mathrm{E}}$ or the extremal luminosity $a L_{\mathrm{E}}$, respectively.

\section{Numerical results}

We have assumed the existence of an accreting system satisfying boundary conditions (2). Although the detailed behaviour is given by the integro-differential nonlinear system (3-5), its essential features can be deduced from the algebraic Eq. (21). The validity of this projection of the original equations to the algebraic problem relies on the existence of appropriate solutions.
We checked numerically that there do exist such solutions and that the relative error made in the above approximations is of the order of $10^{-3}$. Here follows an example. We use dimensionless units with $G=c=1$ and the total mass $M=1$. Appendix B translates relevant quantities into the CGS system that is commonly used in astrophysics.

We assume a relative luminosity $x \equiv \frac{L_{0}}{L_{\mathrm{E}}}=0.1$, the adiabatic index $\Gamma=4 / 3$, the asymptotic speed of sound $a_{\infty}=1 / 50$, the radius of the sphere enclosing the gas $R_{\infty}=10^{6}$ and the surface potential $\phi\left(R_{0}\right)=-0.25$ (that is, the modified size measure $\tilde{R}_{0}=$ 4 ). These quantities in principle suffice to determine a solution; any additional information would lead to a contradiction.

The integro-differential system of Eqs. (1-6) effectively reduces to integro-algebraic equations: Eqs. (1), (5-7) and (12). It has been solved as follows. Choose a parameter $\varrho_{\infty} \approx y /\left(4 \pi R_{\infty}^{3}\right)$ (with some $y$ satisfying the condition $0<y<1$ ). Then, treating $U_{\infty}$ as a free parameter a solution with a sonic point has been constructed. The radius $R_{0}$ of the hard core is found from the condition $\phi\left(R_{0}\right)=-0.25$. It could happen that the mass of the gas $m_{g} \equiv 4 \pi \int_{R_{0}}^{R_{\infty}} r^{2} \varrho \mathrm{d} r$ exceeds 1 ; in such a case the procedure would have to be repeated, with a smaller value of $y$, until the new mass $m_{g}$ becomes smaler than 1 . The mass of the central core is then by definition $M_{\text {core }}=1-m_{g}$. Numerical results confirm that $M_{\text {core }} \approx M_{*}$, as stated in preceding sections.

We have found, in agreement with analytical considerations, that there exist two solutions. Solution I has following characteristics:

i) (sonic point parameters) $R_{*}=556.652, a_{*}^{2}=U_{*}^{2}=$ 0.000797211

ii) ( size and mass of the hard core) $R_{0} \approx 3.95, M_{\text {core }}=$ 0.990456 ;

iii) (asymptotic mass density) $\rho_{\infty}=2.27069 \times 10^{-21}$.

Solution II is characterized by:

i) (sonic point parameters) $R_{*}=61.0175, a_{*}^{2}=U_{*}^{2}=$ 0.000799949;

ii) ( size and mass of the hard core) $R_{0} \approx 0.79, M_{\text {core }}=$ 0.196627 ;

iii) (asymptotic mass density) $\rho_{\infty}=1.912605 \times 10^{-19}$.

The first solution is the test gas approximation, while in the second solution the selfgravitation does matter. In both cases the mass accretion rate $\dot{M}$ is the same. Notice the good quality of the analytic approximation of Sect. 3; this gives sonic point parameters $a_{*}^{2}=U_{*}^{2}=0.0008$, which differs from numerically found values by less than $.4 \%$ for $\sin$ I and less than $0.1 \%$ for $\sin$ II. Figure 2 shows that the normalized mass density $\varrho / \varrho_{\infty} \approx 1+A / R$ for the second solution (with $A=256.032$ and $R>1000$ ); that agrees with one of the analytic estimates obtained in Sect. 3. Figure 3 plots the radial dependence of the ratio $U^{2} /(2 M(R) / R)$. One can clearly see that the infall velocity is significantly bigger and more rapidly asymptotes to $2 M\left(R_{0}\right) / R_{0}$ for solution I than for solution II. This is easy to understand, since the pressure of dense fluids slows down the process of collapse. Both solutions have similar core compactness $2 M\left(R_{0}\right) / R_{0}$. For solution I we have $2 M\left(R_{0}\right) / R_{0} \approx 0.5$ while for solution II $2 m / R_{0}=0.499$. The radiation zone within the gas volume - a region with rapidly changing luminosity $L(R) \approx L_{0} \exp (-1 /(2.5 R))$ - extends outwards from the surface $R_{0}$. Its contribution to the total radiation differs in the two cases, from less than $10 \%$ for $\sin$ I to $40 \%$ for sln II. The redshift for the radiation that comes directly from the surface - found, with good accuracy, from the conservation of energy of individual photons - would be similar in the two 


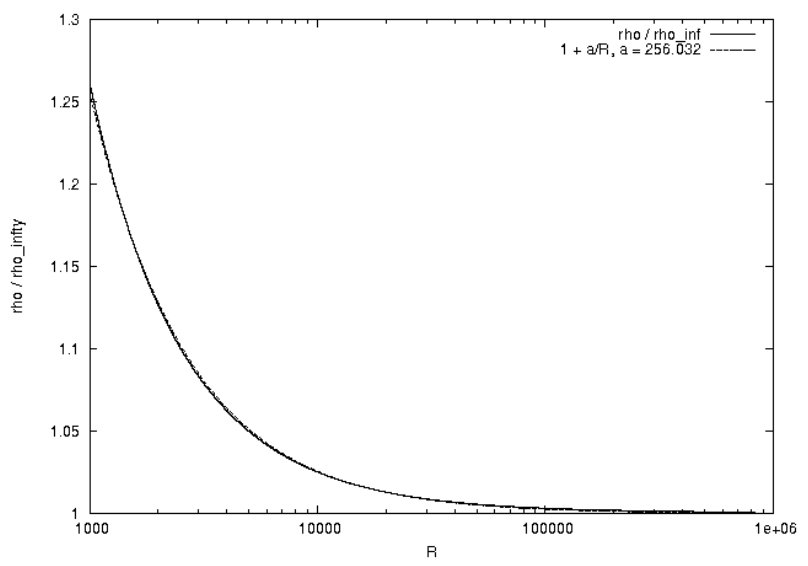

Fig. 2. The normalized mass density $\varrho(R) / \varrho_{\infty}$ (on the ordinate) of sln II in function of the distance $R$ (the abscissa); the graph (solid line) is well approximated by the function $1+256.032 / R$ (broken line).

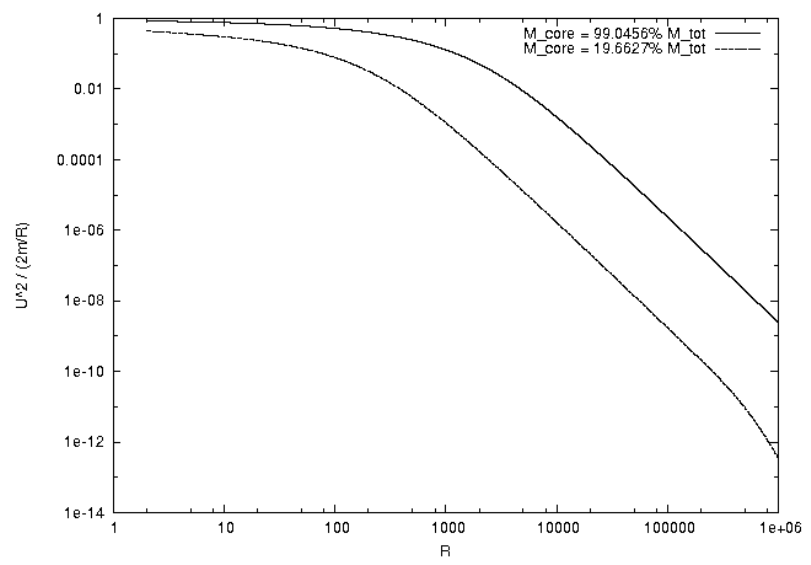

Fig. 3. The infall velocity $U^{2} /(2 M(R) / R)$ of $\sin \mathrm{I}$ (solid line) and sln II (broken line).

cases, but the average redshift and the width of spectral lines would be slightly different.

\section{On the stability of solutions}

The second inequality in our boundary conditions (2) implies the chain of inequalities $8 \pi \rho_{\infty} R_{\infty}^{2} / 3 \leq 2 M / R_{\infty} \leq a_{\infty}^{2}$. Therefore one concludes that $R_{\infty} \ll \lambda_{\mathrm{J}} \equiv \sqrt{3 a_{\infty}^{2} / 8 \pi \rho_{\infty}}$. The quantity $\lambda_{\mathrm{J}}$ is the Jeans length for the accreting configuration. The size of the ball of gas is smaller than $R_{\infty}$ and $R_{\infty}$ is much smaller than the Jeans length $\lambda_{\mathrm{J}}$; therefore one expects that all solutions found in this paper - with test or selfgravitating fluids - are stable. A tentative numerical analysis has been done for spherically symmetric perturbations in the two cases: i) the luminosity is absent; ii) the luminosity and radiation fields are not perturbed. Rachwał (2007) has shown that for integration times that are of the order of the characteristic time $t_{\mathrm{c}} \equiv M / a_{\infty}^{3}$ the evolving perturbations have amplitudes that are roughly constant. A more detailed study of stability in steady accretion will be reported elsewhere.

The next question related to the issue of stability is whether one can justify the assumption - which we make - of stationary accretion in a system where the cloud of accreting gas is heavier than the compact core. In order to solve this problem one should investigate the dynamical version of the Shakura model. The stationary solution of Eqs. (3)-(5) should be inserted as initial data in the dynamical equations and if the dynamical solutions remain close to the stationary one then the assumption of stationarity would be justified. We verified the stationarity of selfgravitating gas in the framework of general relativity (Karkowski et al. 2006; Kinasiewicz et al. 2007). The evolving system remained essentially unchanged in its interior for times of the order of the characteristic time scale $t_{\mathrm{c}}$. The same conclusion is valid for the Shakura model under the simplifying circumstances described in points i) and ii) above.

\section{Final remarks}

We have proven that there exist two radiating systems having the same mass, luminosity, asymptotic temperature and surface potential of the compact component (that is, identical gravitational redshift of radiation emitted from the surface). In the case of low luminosity one of the solutions corresponds to a compact core having a mass close to the total mass surrounded by a test gas; this solution is well known in the literature (Shakura 1974; Okuda \& Sakashita 1977; Maraschi et al. 1978). The alternative is a light compact body enclosed in a heavy cloud of gas; that was not known before. At an extremal point, with the highest luminosity, the two solutions coincide. Only precise measurements of redshift would allow one to distinguish between the two options within the model considered here. Similar conclusions should hold for a stationary wind solution (Marlborough \& René Roy 1970) that can describe the early phase of an exploding system.

Further investigation can focus on including generalrelativistic effects with selfgravitation and/or heating processes (Chan et al. 1979). The general-relativistic analysis of radiating systems reveals main characteristics (including bifurcation) similar to those described above and will be reported elsewhere (Karkowski et al. 2008).

The results of this paper can be applied to a class of subEddington Thorne-Żytkow astrophysical objects, consisting of a compact core (neutron stars or other compact candidates) surrounded by an extended (heavily blown) cloud of gas, which are approximately spherical and Newtonian. By Newtonian systems we mean that the asymptotic speed of sound of the gas is small compared to the speed of light and that the hard core is not very compact, $2 M\left(R_{0}\right) / R_{0} \ll 1$. In most scenarios of formation of TZZS it is believed that their compact core is a small fraction (about one tenth) of the total mass; in such a case the basic Eq. (21) of Sect. 4 gives a relation between observables (total luminosity and the temperature) and the geometry of the system (its size and surface potential at the core). In particular, if one assumes that the core is a neutron star (and thus the mass is essentially known), then by reversing the reasoning of this paper one can estimate the mass of the total system.

The generalized Shakura model offers several analytic predictions: the luminosity is given analytically, two parameters ( $U$ and $a$ ) of a sonic point are the same as in a Bondi model and bifurcating solutions should emerge. These can be compared with the outcome of numerical simulations. Therefore this model offers a useful test for numerical codes.

Acknowledgements. This paper has been partially supported by the MNII grant 1PO3B 01229. We thank Bogusz Kinasiewicz and Zdobysław Świerczyński for their help in performing the numerical calculations. K.R. thanks the Foundation for Polish Science for financial support. 


\section{Appendix A: Proof of the theorem}

The two extremality conditions $F=0, \partial_{y} F(x, y)=0$ (see Theorem in the main text) yield two equations

$a-\beta(1-b)(b-a \exp (-a \gamma))^{2}=0$,

$b-a \exp (-a \gamma)=2(1-b)$.

From Eq. (A.1) one obtains the desired expressions

$b=\frac{2+a \exp (-a \gamma)}{3}, \quad a=4 \beta(1-b)^{3}$.

Inserting the second of Eq. (A.2) into the second equation in (A.1), one arrives at

$b=\frac{2}{3}+\frac{4 \beta}{3}(1-b)^{3} \exp \left(-4 \beta(1-b)^{3} \gamma\right)$.

Both sides of this equation are continuous functions of $b$ and at $b=0$ the right hand side of Eq. (A.3) is greater than the left hand side, while at $b=1$ the opposite holds true. Therefore there exists a solution. Closer inspection shows that there is only one extremal solution. This fact guarantees that solutions $y(x)$ that bifurcate from $(a, b)$ extend into the whole interval $x \in(0, a)$. (Hint: use the implicit function theorem - see Sect. III.8 in (Schwarz 1967) - and the fact that $(a, b)$ is the unique extremal point). The specific form of a solution close to an extremal point can be obtained as follows. Insert $x=a+\epsilon, y=b+y(\epsilon)$ into $F(x, y)=0$ and expand $F$ keeping the terms of lowest order. One obtains a reduced algebraic equation

$$
\begin{gathered}
\left(1+2 \beta(1-b)\left(b-a \mathrm{e}^{-a \gamma}\right)\left(a \mathrm{e}^{-a \gamma} \gamma-\mathrm{e}^{-a \gamma}\right)\right) \epsilon \\
+\beta\left(3 b-1-2 a \mathrm{e}^{-a \gamma}\right) y^{2}(\epsilon)=0 .
\end{gathered}
$$

Notice that at the extremal point $3 b-1-2 a \exp (-a \gamma)=1-$ $a \exp (-a \gamma)$ (see the second of Eqs. (A.1)) while

$$
\begin{gathered}
2 \beta(1-b)(b-a \exp (-a \gamma))(a \exp (-a \gamma) \gamma-\exp (-a \gamma))= \\
2 a(a \exp (-a \gamma) \gamma-\exp (-a \gamma)) /(b-a \exp (-a \gamma) .
\end{gathered}
$$

Inserting Eq. (A.5) into Eq. (A.4) and finding $y(\epsilon)$ from the latter leads to the approximate solution of ii).

Now we shall prove the third part of the Theorem. Let $\left(x_{0}, y_{0}\right)$ be a non-extremal solution of $F(x, y)=0$ with the domain being subset $x \mathrm{e}^{-x}<y$ of the square $0<x<1,0<y<1$. Then from the implicit function theorem there exists a curve $x(y)$ such that $F(x(y), y)=0$ for $y$ belonging to some vicinity of $y_{0}$. Along this curve one has

$$
\frac{\mathrm{d} x}{\mathrm{~d} y}=\beta \frac{\left(y-x \mathrm{e}^{-x \gamma}\right)\left(3 y-2-x \mathrm{e}^{-x \gamma}\right)}{1+2 \beta(1-y)\left(y-x \mathrm{e}^{-x \gamma}\right)\left(\mathrm{e}^{-x \gamma}-\gamma x \mathrm{e}^{-x \gamma}\right)} .
$$

The denominator is strictly positive while the numerator vanishes only at extremal points. That proves the assertion. It follows from the form of the approximate solution constructed in part ii) that $x=a$ is the extremal value of the relative luminosity.

\section{Appendix B: Numerical data}

We present numerical data of Sect. 5 in standard astrophysical units. Let the total mass be given in the units of solar mass $M_{0}=1.989 \times 10^{33} \mathrm{~g}, M=M_{0}\left(M / M_{)}\right)$. Then $L_{\mathrm{E}}=$ $1.3 \times 10^{38}\left(M / M_{0}\right) \mathrm{erg} / \mathrm{s}$ and for $x=0.1$ we have $L_{0}=1.3 \times$ $10^{37}\left(M / M_{0}\right)$. The asymptotic speed of sound is $a_{\infty}=c / 50=$ $6 \times 10^{8} \mathrm{~cm} / \mathrm{s}$, the radius of the sphere enclosing the gas is $R_{\infty}=1.5 \times 10^{11}\left(M / M_{0}\right) \mathrm{cm}$ and the surface potential is $\phi\left(R_{0}\right)=$ $-0.25 c^{2}=-2.25 \times 10^{20} \mathrm{~cm}^{2} / \mathrm{s}^{2}$. The modified size measure is now $\tilde{R}_{0}=6 \times 10^{5}\left(M / M_{0}\right) \mathrm{cm}$.

The two solutions have the following characteristics:

A. Solution I.

i) (sonic point parameters) $R_{*}=8.35 \times 10^{7}\left(M / M_{0}\right) \mathrm{cm}$, $a_{*}=\left|U_{*}\right|=8.46 \times 10^{8} \mathrm{~cm} / \mathrm{s}$;

ii) (size and mass of the hard core) $R_{0}=5.93 \times$ $10^{5}\left(M / M_{0}\right) \mathrm{cm}, M_{\text {core }}=1.98 \times 10^{33}\left(M / M_{0}\right) g$;

iii) (asymptotic mass density) $\rho_{\infty} \approx 6 \times 10^{-4}\left(M_{0} / M\right)^{2} \mathrm{~g} / \mathrm{cm}^{3}$.

B. Solution II.

i) (sonic point parameters) $R_{*}=9,15 \times 10^{6}\left(M / M_{0}\right) \mathrm{cm}$, $a_{*}=\left|U_{*}\right|=8.46 \times 10^{8} \mathrm{~cm} / \mathrm{s}$

ii) (size and mass of the hard core) $R_{0}=1.18 \times$ $10^{5}\left(M / M_{0}\right) \mathrm{cm}, M_{\text {core }}=3,92 \times 10^{32}\left(M / M_{0}\right) g$;

iii) (asymptotic mass density) $\rho_{\infty} \approx 1.1 \times$ $10^{-1}\left(M_{0} / M\right)^{2} \mathrm{~g} / \mathrm{cm}^{3}$.

\section{References}

Abramowicz, M., Kluźniak, W., \& Lasota, J.-P. 2002, A\&A, 396, L31 Bondi, H. 1952, MNRAS, 112, 195

Broderick, A. E., \& Narayan, R. 2006, ApJ, 638, L21

Buff, J., \& McCray, J. 1974, ApJ, 189, 147

Chan, K. L., Henriksen, R. N., \& Chau, W. Y. 1979, A\&A, 75, 133

Chevalier, R. A. 1993, ApJ, 411, L33

Chia, T. T. 1978 , MNRAS, 185, 561

Chia, T. T. 1979 , MNRAS, 188,75

Grindlay, J. E. 1978, ApJ, 221, 234

Karkowski, J., Kinasiewicz, B., Mach, P., Malec, E., \& Świerczyński, Z. 2006, Phys. Rev. D, 73, 021503(R)

Karkowski, J., Malec, E., Roszkowski, K., \& Świerczyński, Z. 2008,

in preparation

Kinasiewicz, B., Mach, P., \& Malec, E. 2007, Int. J. Geom. Meth. Mod. Phys., 4, 197

Maraschi, L., Treves, A., \& Reina, C. 1978, A\&A, 66, 99

Marlborough, J. M., \& René Roy, J. 1970, ApJ, 160, 221

Mazur, P. O., \& Mottola, E. 2004, Proc. Nat. Acad. Sci., 101, 9545

Okuda, T., \& Sakashita, S. 1977, Ap\&SS, 52, 35

Park, M.-G., \& Miller, G. S. 1991, ApJ, 371, 708

Rachwał, B. 2007, Master's thesis, Institute of Physics, Jagiellonian University

Rezzolla, L., \& Miller, J. C. 1994, Class. Quantum Grav., 11, 1815

Schwarz, L. 1967, Analyse Mathématique (Paris: Hermann)

Shakura, N. I. 1974, AZh, 51, 441

Thorne, K. S., \& Żytkow, A. N. 1977, ApJ, 212, 832

Thorne, K. S., Flammang, R. A., \& Żytkow, A. N. 1981, MNRAS, 194, 475 